\title{
SKEW-SYMMETRIC ELEMENTS OF RATIONAL GROUP ALGEBRAS
}

\author{
DISHARI CHAUDHURI
}

\begin{abstract}
Let $R G$ be the group ring of a finite group $G$ over a commutative ring $R$ with 1 . An element $x$ in $R G$ is said to be skew-symmetric with respect to an involution $\sigma$ of $R G$ if $\sigma(x)=-x$. A structure theorem for the skew-symmetric elements of $F G$ is given where $F$ is an algebraic extension of $\mathbb{Q}$ which generalizes some previously known results in this direction.
\end{abstract}

\section{INTRODUCTION}

Let $R G$ be the group ring of a finite group $G$ over a commutative ring $R$ with 1 and let $\sigma$ be an involution of $R G$. That is, $\sigma: R G \rightarrow R G$ is such that for $x, y \in R G, \sigma$ satisfies the following conditions: (i) $\sigma(x+y)=\sigma(x)+\sigma(y)$, (ii) $\sigma(x y)=\sigma(y) \sigma(x)$ and (iii) $\sigma^{2}(x)=x$. Let $R G_{\sigma}^{+}=\{\gamma \in R G \mid \sigma(\gamma)=\gamma\}$ and $R G_{\sigma}^{-}=\{\gamma \in R G \mid \sigma(\gamma)=-\gamma\}$ be the set of symmetric and skew-symmetric elements of $R G$ respectively with respect to the involution $\sigma$. If $\sigma$ is an $R$-linear extension of an involution of $G$, then $R G_{\sigma}^{-}$is generated by $\left\{g-\sigma(g) \mid g \in G \backslash G_{\sigma}\right\} \cup\left\{r g \mid r \in R_{2}, g \in G_{\sigma}\right\}$ as an $R$-module, where $G_{\sigma}=\{g \in G \mid \sigma(g)=g\}$ and $R_{2}=\{r \in R \mid 2 r=0\}$. Note that $R G_{\sigma}^{-}$may not be a subring of $R G$ in general. Now, $R G$ may be viewed as a Lie ring with the help of the bracket operation $[x, y]=x y-y x$ for $x, y \in R G$. Then, $R G_{\sigma}^{-}$becomes a Lie subring of $R G$. There are some strong relations between the structure of $R G_{\sigma}^{-}$and $R G$ as Lie rings which have been studied by a few authors (for example, Ami69, [ZS81, [GS93]). Also there are some close relations between polynomial identities satisfied by the unitary units of $R G$ and the Lie algebra $R G_{\sigma}^{-}$([GM03]). The question of when $R G_{\sigma}^{-}$is commutative has been completely answered in terms of the group elements of $G$ ([JM05,

1991 Mathematics Subject Classification. Primary 16S34; Secondary 16W10.

Key words and phrases. Group Rings, Involution, Skew-Symmetric Elements, Lie ring, Central Simple Algebras.

The author was supported by DAE (Government of India) and National Board for Higher Mathematics with reference number 2/40(16)/2016/ R\&D-II/5766 during this project. The author would like to thank IISER Mohali for providing good research facilities when this project was carried out. The author is very grateful to Abhay Soman for many insightful discussions. Finally the author would like to thank the unknown referee whose valuable comments and suggestions helped a great deal in improving the exposition of this article. 
[JM06], [CM07], [CJMM09]). Lie properties of $R G_{\sigma}^{-}$has been studied as well ([LSS09]). So we can see that the Lie algebra $R G_{\sigma}^{-}$has been a useful object of study in quite a few cases. Hence it is interesting to investigate the structure of $R G_{\sigma}^{-}$itself.

Given a semisimple group algebra $R G$, the Lie algebra $R G_{\sigma}^{-}$will be semisimple and hence it is natural to seek a concrete description of the simple Lie algebras into which it decomposes as a direct sum. When $R=\mathbb{C}$ and $\sigma$ is the canonical involution of $\mathbb{C} G$, that is, the $\mathbb{C}$-linear extension of $g \mapsto g^{-1}$, the structure of $\mathbb{C} G_{\sigma}^{-}$is given as a direct sum of classical simple Lie algebras by Cohen and Taylor [CT07. The Lie algebra $\mathfrak{g l}(n)$ is the space of all linear transformations of $\mathbb{C}^{n}$, where the Lie product is the usual bracket operation. If $n \geq 1$ and if $b$ is a non-degenerate alternating or symmetric bilinear form on $\mathbb{C}^{n}$, then the subspace of $\mathfrak{g l}(n)$ consisting of all $x$ such that $b(x u, v)+b(u, x v)=0$ for all $u, v \in \mathbb{C}^{n}$ is a Lie algebra. When $b$ is skew-symmetric, $n$ is necessarily even, and we have the symplectic Lie algebra $\mathfrak{s p}(n)$. When $b$ is symmetric, we have the orthogonal Lie algebra $\mathfrak{o}(n)$. Let $\chi$ be a character of $G$. It was shown in [CT07] that $\mathbb{C} G_{\sigma}^{-}$admits the Lie algebra decomposition:

$$
\mathbb{C} G_{\sigma}^{-} \simeq \bigoplus_{\chi \in \mathfrak{R}} \mathfrak{o}(\chi(1)) \oplus \bigoplus_{\chi \in \mathfrak{S p}} \mathfrak{s p}(\chi(1)) \oplus \bigoplus_{\chi \in \mathfrak{C}}^{\prime} \mathfrak{g l}(\chi(1))
$$

where $\mathfrak{R}, \mathfrak{S p}$ and $\mathfrak{C}$ are the sets of irreducible characters of $G$ of real, symplectic and complex types, respectively. The prime signifies that there is just one summand $\mathfrak{g l}(\chi(1))$ for each pair $\{\chi, \bar{\chi}\}$ from $\mathfrak{C}$. A slightly more general setting has been taken into consideration by Marin [Mar10], where $R=\mathbb{K}$, a field of characteristic zero such that each ordinary representation of $G$ is defined over $\mathbb{K}$ (for instance, any $\mathbb{K}$ containing the field of cyclotomic numbers) and $\sigma$ is the $\mathbb{K}$-linear extension of the map $g \mapsto \alpha(g) g^{-1}$, where $\alpha: G \rightarrow \mathbb{K}^{\times}$is a multiplicative character of $G$. Here also $\mathbb{K} G_{\sigma}^{-}$is shown to admit a similar kind of decomposition. Both the papers have used Frobenius-Schur Theory and concluded an analogue of Wedderburn decomposition for the corresponding Lie subalgebras. All these results motivated us to study these kinds of Lie algebras on a more general setting. We have considered the group algebra $F G$ of a finite group $G$ where $F$ is an algebraic extension of $\mathbb{Q}$ and its Lie subalgebra $F G_{\sigma}^{-}$with respect to any arbitrary involution $\sigma$ of $F G$.

As a result of this study we have obtained the following results. All the notions of the statement will be recalled in section 2 . 
Theorem 1.1. Let $F G$ be a group algebra of a finite group $G$ over a field $F$ which is an algebraic extension of $\mathbb{Q}$ and $\sigma$ be an involution of $F G$. Then there exists an involution $\theta$ on $F$ and a right $F$-vector space $M$ which is also a left $F G$-module and a nonsingular hermitian or skew-hermitian form $h: M \times M \rightarrow F$ on $M$ (with respect to the involution $\theta$ on $F$ ) such that $\sigma$ is the adjoint involution of $h$ and

$$
F G_{\sigma}^{-}=\{f \in F G \mid h(f(x), y)+h(x, f(y))=0 \text { for all } x, y \in M\} .
$$

The $\theta$ and $M$ in the above statement have been constructed explicitly in this work (Section 3). Our second main result is a decomposition theorem for the above Lie algebra of skew-symmetric elements.

Theorem 1.2. If $F G \simeq \bigoplus_{V \in \tilde{G}} \operatorname{End}_{D}(V)$ as F-algebras, where $\tilde{G}$ denotes the set of all irreducible left $F G$-modules up to isomorphism with $D$ being division algebras over $F$ and the degree of the central simple algebra $\operatorname{End}_{D}(V)$ over its center $Z$ is $n_{V}$, then

$$
F G_{\sigma}^{-} \simeq \bigoplus_{V \in \tilde{G}_{o}} \mathfrak{o}(V) \oplus \bigoplus_{V \in \tilde{G}_{s p}} \mathfrak{s p}(V) \oplus \bigoplus_{V \in \tilde{G}_{u}} \mathfrak{g} \mathfrak{l}(V)
$$

as Lie algebras over $F$ where $\tilde{G}_{o}, \tilde{G}_{s p}$ and $\tilde{G}_{u}$ are subsets of $\tilde{G}$ such that

$$
\begin{array}{r}
\tilde{G}_{o}=\left\{V \mid \sigma\left(\operatorname{End}_{D}(V)\right)=\operatorname{End}_{D}(V), \operatorname{dim}_{Z}\left(\operatorname{End}_{D}(V)\right)_{\sigma}^{-}=n_{V}\left(n_{V}-1\right) / 2\right\}, \\
\tilde{G}_{s p}=\left\{V \mid \sigma\left(\operatorname{End}_{D}(V)\right)=\operatorname{End}_{D}(V), \operatorname{dim}_{Z}\left(\operatorname{End}_{D}(V)\right)_{\sigma}^{-}=n_{V}\left(n_{V}+1\right) / 2,\right. \\
\left.n_{V} \text { is even }\right\}, \\
\tilde{G}_{u}=\left\{V \mid \sigma\left(\operatorname{End}_{D}(V)\right)=\operatorname{End}_{D}(V) \text { or } \sigma\left(\operatorname{End}_{D}(V)\right)=\operatorname{End}_{D^{o p}}\left(V^{o p}\right),\right. \\
\left.\operatorname{dim}_{Z}\left(\operatorname{End}_{D}(V)\right)_{\sigma}^{-}=n_{V}^{2}\right\} .
\end{array}
$$

The prime signifies that there is just one summand $\mathfrak{g l}(V)$ for each pair $\left\{V, V^{o p}\right\}$ from $\tilde{G}$.

The manuscript has been divided into three sections. The second section recalls some basic definitions and results to be used in Section 3. The third section is devoted to the proof of the main theorems, where we will first decompose $F G$ as a semisimple algebra with involution into a direct product of central simple algebras with involution. On each such central simple algebra, we will construct a nonsingular hermitian or skew hermitian form with the help of its involution. This nonsingular form will give rise to a Lie algebra on that component which we will show to be exactly equal to the image of the Lie algebra of skew-symmetric elements of $F G$ under the projection of $F G$ onto that component. Finally we will extend the results similarly to the whole of $F G$. 


\section{BACKGROUND}

Let the pair $(A, \sigma)$ denote an algebra $A$ with 1 over a field $F$ with $\sigma$ being an involution on $A$ and is such that $F=\mathcal{Z}(A) \cap A_{\sigma}^{+}$, where $\mathcal{Z}(A)$ is the center of the algebra $A$. We will assume throughout this section that char $F \neq 2$ and algebras are always finite dimensional over the corresponding fields. Note that the map $\sigma$ preserves the center $\mathcal{Z}(A)$. For a commutative algebra $K$ over $F$, we set $[K: F]=\operatorname{dim}_{F} K$.

As the involution $\sigma$ is an anti-automorphism with $\sigma^{2}=i d$, the restriction of $\sigma$ to $\mathcal{Z}(A)$ is an automorphism which is either the identity or of order 2. Throughout this paragraph we will assume that $(A, \sigma)$ is an $F$-algebra with involution $\sigma$ such that $A$ has no non-trivial two sided ideal $I$ with $\sigma(I)=I$. With this assumption, either $\mathcal{Z}(A)=F$ or $\mathcal{Z}(A)$ is a quadratic étale extension of $F$ (which means that $\mathcal{Z}(A)$ is either a field which is a separable quadratic extension of $F$ or $\mathcal{Z}(A) \simeq F \times F)$. One can refer to the beginning of Section 3, BGBT18] for this paragraph. One says that $(A, \sigma)$, or the involution $\sigma$, is of the first kind or the second kind, respectively, according to whether $[\mathcal{Z}(A): F]$ equals 1 or 2 . As long as the center $\mathcal{Z}(A)$ of $A$ is a field, it follows that $A$ is central simple as a $\mathcal{Z}(A)$-algebra. This follows from the observation that if $I$ is a two sided ideal of $A$, then the two sided ideal $I+\sigma(I)$ is invariant under $\sigma$. Thus $I+\sigma(I)=0$ according to the assumption in the beginning of this paragraph. This implies that $\sigma(I)=-I=I$, which in turn implies that $I=0$. Thus $A$ has no non-trivial two sided ideal and hence is simple. However, if $(A, \sigma)$ is of the second kind, then as $[\mathcal{Z}(A): F]=2$, we may also have $\mathcal{Z}(A)=F \times F$.

Involutions of the first kind can be divided into two categories. Let $(A, \sigma)$ be a central simple $F$-algebra of degree $n$ with involution of the first kind and let $L$ be a splitting field of $A$ (that is, $L$ is a field containing $F$ such that $\left.A_{L}=A \otimes_{F} L \simeq M_{n}(L)\right)$. Then $\sigma$ extends to an involution of the first kind $\sigma_{L}=\sigma \otimes I d_{L}$ on $A_{L}$. Let $V$ be an $L$-vector space of dimension $n$. There is a nonsingular symmetric or skew-symmetric bilinear form $b$ on $V$ and $\left(A_{L}, \sigma_{L}\right) \simeq\left(\operatorname{End}_{L}(V), \sigma_{b}\right)$, where $\sigma_{b}$ is the adjoint involution (for the definition of adjoint involution see Lemma 2.5 below) of the form $b$. (Proposition 2.1, KMRT98]). If $b$ is symmetric, we say the involution $\sigma$ is of orthogonal type. If $b$ is skew-symmetric, then $\sigma$ is said to be of symplectic type. As a parallel terminology involutions of the second kind are also sometimes said to be of unitary type.

The following definition is 1.2.7 of [Knu91]. 
Definition 2.1. For any $\operatorname{ring} R$, let $R^{o p}$ be the opposite ring, that is, the same additive group with reversed multiplication

$$
a^{o p} \cdot b^{o p}=(b a)^{o p}
$$

where $a^{o p}$ stands for $a$ as an element of $R^{o p}$. Involutions on $R$ are isomorphisms $\phi$ : $R \rightarrow R^{o p}$ of rings such that $\phi^{2}=1_{R}$. For any ring $R$, the product $R \times R^{o p}$ has an involution

$$
\left(a, b^{o p}\right) \mapsto\left(b, a^{o p}\right)
$$

This product is called the hyperbolic ring of $R$ and is denoted by $H(R)$. This involution is called the exchange involution.

The result below follows from Proposition 2.14 and its proof in [KMRT98. Some of the notations used are from definition 2.1 above.

Lemma 2.2. Let $A$ be a semisimple F-algebra of the form $A_{1} \times A_{2}$, where $A_{1}, A_{2}$ are central simple $F$-algebras and $A$ is endowed with an involution $\sigma$ of the second kind. Then $(A, \sigma)=\left(A_{1} \times A_{2}, \sigma\right) \simeq\left(A_{1} \times A_{1}{ }^{o p}, \varepsilon\right)$ where $\varepsilon$ is the exchange involution. That is, $(A, \sigma) \simeq\left(H\left(A_{1}\right), \varepsilon\right)$.

The following is example 1.2.8 of Chapter $I$ of [Knu91].

Proposition 2.3. Let $A$ be a semisimple F-algebra with involution $\sigma$. We can decompose $A$ as a product $A_{1} \times \cdots \times A_{n} \times B_{1} \times \cdots \times B_{m}$, where the $A_{i}$ are simple and $\sigma\left(A_{i}\right)=A_{i}$ and the $B_{j}$ are products of two simple algebras $A_{j}^{\prime} \times A_{j}^{\prime \prime}$ with $\sigma\left(A_{j}^{\prime}\right)=A_{j}^{\prime \prime}$. The rings $B_{j}$ are isomorphic to hyperbolic rings, $B_{j} \simeq H\left(A_{j}^{\prime}\right)$.

Proof. The proof follows from the fact that $\sigma$ being an involution, when restricted to any one of the simple components in the Wedderburn decomposition of the semisimple algebra $A$, will either take that component to itself or to another simple component due to simplicity of the domain. Thus by rearranging the simple components in the Wedderburn decomposition of $A$ according to whether $\sigma$ sends the simple component to itself or to another simple component, we get the decomposition as mentioned in the statement. Clearly, $\sigma$ restricted to $B_{j}$ 's are involutions of the second kind where the center $\mathcal{Z}\left(B_{j}\right)=\mathcal{Z}\left(A_{j}^{\prime}\right) \times \mathcal{Z}\left(A_{j}^{\prime \prime}\right)$ is a product of two factors $K \times K$ where $K$ is a finite field extension of $F$. The fact that $B_{j} \simeq H\left(A_{j}^{\prime}\right)$ follows from Lemma 2.2.

Let us recall the definition of a hermitian form with respect to an involution on a finite dimensional central simple algebra. (See 4.A, [KMRT98]). 
Definition 2.4 (Hermitian Forms). Let $E$ be a central simple algebra over a field $F$ with char $F \neq 2$ and let $M$ be a finitely generated right $E$-module. Suppose that $\theta: E \rightarrow E$ is an involution (of any kind) on $E$. A hermitian form on $M$ (with respect to the involution $\theta$ on $E$ ) is a bi-additive map $h: M \times M \rightarrow E$ such that:

(1) $h(x \alpha, y \beta)=\theta(\alpha) h(x, y) \beta$ for all $x, y \in M$ and $\alpha, \beta \in E$,

(2) $h(y, x)=\theta(h(x, y))$ for all $x, y \in M$.

If (2) is replaced by $h(y, x)=-\theta(h(x, y))$ for all $x, y \in M$, the form $h$ is called skew-hermitian. If $E=F$ and $\theta=I d$, hermitian (resp. skew-hermitian) forms are the symmetric (resp. skew-symmetric) bilinear forms on the finite dimensional vector space $M$ over $F$.

The hermitian or skew-hermitian form $h$ on the right $E$-module $M$ is called nonsingular if the only element $x \in M$ such that $h(x, y)=0$ for all $y \in M$ is $x=0$.

The following is Proposition 4.1 in [KMRT98. The notations used are from the above definition 2.4 .

Lemma 2.5 (Adjoint Involution). For every nonsingular hermitian or skew-hermitian form $h$ on $M$, there exists a unique involution $\sigma_{h}$ on $\operatorname{End}_{E}(M)$ such that $\sigma_{h}(\alpha)=\theta(\alpha)$ for all $\alpha \in F$ and

$$
h(x, f(y))=h\left(\sigma_{h}(f)(x), y\right) \text { for } x, y \in M, f \in \operatorname{End}_{E}(M) .
$$

The involution $\sigma_{h}$ is called the adjoint involution with respect to $h$.

Let $Z / F$ be a finite extension of fields, $E$ a central simple algebra over $Z$ and $T$ a central simple $F$-algebra contained in $E$. Suppose that $\theta$ is an involution (of any kind) on $E$ which preserves $T$.

Definition 2.6 (Involution trace). An F-linear map $s: E \rightarrow T$ is called an involution trace if it satisfies the following conditions:

(1) $s\left(t_{1} x t_{2}\right)=t_{1} s(x) t_{2}$ for all $x \in E$ and $t_{1}, t_{2} \in T$;

(2) $s(\theta(x))=\theta(s(x))$ for all $x \in E$;

(3) if $x \in E$ is such that $s(\theta(x) y)=0$ for all $y \in E$, then $x=0$.

Example 1 (Reduced Trace). In the above definition 2.6, if $T=F=Z$, the reduced trace $\operatorname{Trd}_{E / F}: E \rightarrow F$ is an involution trace. The first two conditions follow from 2.2 and 2.16 in KMRT98 and the third condition follows from the fact that the bilinear 
reduced trace form $T_{E / F}: E \times E \rightarrow F$ defined by: $T_{E / F}(x, y)=\operatorname{Trd}_{E / F}(x y)$ for $x, y \in E$ is nonsingular (Theorem 9.9, [Rei03]).

Example 2 (Linear Forms). In definition 2.6, if $E=Z$ and $T=F$, every nonzero linear map $l: Z \rightarrow F$ which commutes with $\theta$ is an involution trace. Nonzero linear forms $Z \rightarrow F$ always exist if the extension $Z / F$ is separable. For example, $\operatorname{Tr} d_{Z / F}$ will be an involution trace (that is, nonzero linear form) for the trivial involution if and only if $Z / F$ is a separable field extension ([Knu91, Chapter $I, 7.3 .2$ ).

\section{Proof of the MAIN THEOREMS}

We begin with the Wedderburn Artin decomposition of $F G$ with involution.

Proposition 3.1. Let $G$ be a finite group and $\sigma$ be an involution of $F G$, where $F$ is an algebraic extension of $\mathbb{Q}$. Then as a semisimple algebra with involution, $F G$ decomposes into:

$$
\begin{array}{r}
(F G, \sigma) \simeq \quad\left(M_{a_{1}}\left(D_{1}\right), \sigma_{a_{1}}\right) \times \cdots \ldots \ldots \times\left(M_{a_{r}}\left(D_{r}\right), \sigma_{a_{r}}\right) \times \\
\left(M_{b_{1}}\left(D_{1}^{\prime}\right) \times M_{b_{1}}\left(D_{1}^{\prime}\right)^{o p}, \sigma_{b_{1}}\right) \times \cdots \ldots \ldots \ldots \ldots \\
\cdots \times\left(M_{b_{t}}\left(D_{t}^{\prime}\right) \times M_{b_{t}}\left(D_{t}^{\prime}\right)^{o p}, \sigma_{b_{t}}\right)
\end{array}
$$

where $\sigma_{a_{i}}$ are involutions of $M_{a_{i}}\left(D_{i}\right)$ and $\sigma_{b_{j}}$ are involutions of $M_{b_{j}}\left(D_{j}^{\prime}\right) \times M_{b_{j}}\left(D_{j}^{\prime}\right)^{\text {op }}$ such that $\sigma_{b_{j}}\left(M_{b_{j}}\left(D_{j}^{\prime}\right) \times\{0\}\right)=\{0\} \times M_{b_{j}}\left(D_{j}^{\prime}\right)^{\text {op }}$, for $1 \leq i \leq r$ and $1 \leq j \leq t$.

Proof. As FG is semisimple, by Wedderburn Structure Theorem, $F G$ is isomorphic as $F$-algebra to a product of simple algebras in the following way:

$$
F G \simeq M_{n_{1}}\left(D_{1}\right) \times M_{n_{2}}\left(D_{2}\right) \times \cdots \times M_{n_{k}}\left(D_{k}\right)
$$

where $D_{i}$ are finite dimensional division algebras over $F$ such that the center $\mathcal{Z}\left(M_{n_{i}}\left(D_{i}\right)\right)=$ $\mathcal{Z}\left(D_{i}\right)=\mathcal{Z}_{i}$ is a finite field extension over $F$. Thus $M_{n_{i}}\left(D_{i}\right)$ are central simple algebras over $\mathcal{Z}_{i}$. Now, by Proposition 2.3, $(F G, \sigma)$ decomposes as $F$-algebra into

$$
\begin{aligned}
F G \simeq & M_{a_{1}}\left(D_{1}\right) \times \cdots \cdots \times M_{a_{r}}\left(D_{r}\right) \times M_{b_{1}}\left(D_{1}^{\prime}\right) \times M_{b_{1}}\left(D_{1}^{\prime \prime}\right) \\
& \times \cdots \cdots \times M_{b_{t}}\left(D_{t}^{\prime}\right) \times M_{b_{t}}\left(D_{t}^{\prime \prime}\right),
\end{aligned}
$$

where $\sigma\left(M_{a_{i}}\left(D_{i}\right)\right)=M_{a_{i}}\left(D_{i}\right)$ and $\sigma\left(M_{b_{j}}\left(D_{j}^{\prime}\right)\right)=M_{b_{j}}\left(D_{j}^{\prime \prime}\right)$, for $1 \leq i \leq r, 1 \leq j \leq t$. Again, by Proposition 2.3 ,

$$
\left(M_{b_{j}}\left(D_{j}^{\prime}\right) \times M_{b_{j}}\left(D_{j}^{\prime \prime}\right), \sigma\right) \simeq\left(M_{b_{j}}\left(D_{j}^{\prime}\right) \times M_{b_{j}}\left(D_{j}^{\prime}\right)^{o p}, \sigma_{b_{j}}\right)
$$

where $\sigma_{b_{j}}$ is the switch involution. Taking $\sigma$ restricted to $M_{a_{i}}\left(D_{i}\right)$ as $\sigma_{a_{i}}$ for $1 \leq i \leq r$, the result follows. 
All the constructions and computations henceforth will be written down for the component $M_{n_{i}}\left(D_{i}\right)$ only in the Wedderburn decomposition of $F G$ as they are similar for the direct product $M_{b_{j}}\left(D_{j}^{\prime}\right) \times M_{b_{j}}\left(D_{j}^{\prime}\right)^{o p}$.

Let us fix $i$ and denote $n_{i}, D_{i}$ and $\sigma_{n_{i}}$ as $n, D$ and $\sigma$ respectively. We concentrate on $\left(M_{n}(D), \sigma\right)$. Now, $M_{n}(D)$ is isomorphic as $F$-algebra to $\operatorname{End}_{D}\left(D^{n}\right)$, where $D^{n}$ can be viewed as a right vector space over $D$. As $M_{n}(D)$ is Brauer equivalent to $D$, by a well known theorem by A.A. Albert ([KMRT98], Theorem 3.1), there exists an involution, say $\theta$ on $D$ which is of the same kind as $\sigma$. Thus, according to Theorem 4.2, [KMRT98, there exists a nonsingular hermitian or skew-hermitian form $h: D^{n} \times D^{n} \rightarrow D$ with respect to $\theta$ such that $\sigma$ is the adjoint involution of $h$ (recall definition 2.5), that is, $\sigma=\sigma_{h}$ and it satisfies $h(x, f(y))=h\left(\sigma_{h}(f)(x), y\right)$ for all $x, y \in D^{n}$ and $f \in \operatorname{End}_{D}\left(D^{n}\right)$.

Let $Z$ denote the center of $D$. Then $D$ is a finite dimensional central simple algebra over $Z$ which is a finite field extension over $F$ and $F$ again is an algebraic extension of $\mathbb{Q}$. Hence $Z / F$ is separable. By definition, $\theta$ fixes $F$ pointwise. Thus we can choose a nonzero linear form $l: Z \rightarrow F$ (by Example 2) that commutes with $\theta$ restricted to $Z$. Let $\operatorname{Trd}_{D / Z}: D \rightarrow Z$ be the reduced trace of the central simple algebra $D$ over $Z$ which is an involution trace by Example 1. Now take the composition of these two maps to define the map $s: D \rightarrow F$ where $s=l \circ \operatorname{Tr} d_{D / Z}$.

Lemma 3.2. With the notations as above, $s$ is an involution trace.

Proof. We check the conditions one by one.

(1) $s\left(\alpha_{1} x \alpha_{2}\right)=\left(l \circ \operatorname{Trd}_{D / Z}\right)\left(\alpha_{1} x \alpha_{2}\right)=\alpha_{1} s(x) \alpha_{2}$ for all $\alpha_{1}, \alpha_{2} \in F \subseteq Z$ and $x \in D$ follows directly from the definition of $l$ and $\operatorname{Trd}_{D}$.

(2) $s(\theta(x))=\left(l \circ \operatorname{Trd}_{D / Z}\right)(\theta(x))=l\left(\theta\left(\operatorname{Tr}_{D / Z}(x)\right)\right)=\theta\left(l\left(\operatorname{Tr}_{D / Z}(x)\right)\right)=\theta(s(x))$ for all $x \in D$ as $\operatorname{Trd}_{D / Z}$ is an involution trace and $l$ is a nonzero linear form that commutes with $\theta$ restricted to $Z$.

(3) Let $x \in D$ be such that $s(\theta(x) y)=0$ for all $y \in D$. This means $l\left(\operatorname{Tr}_{D / Z}(\theta(x) y)\right)=$ 0 for all $y \in D$. Since $l$ is a nonzero linear form on a finite dimensional vector space $Z$ over $F$, we must have $\operatorname{Tr}_{D / Z}(\theta(x) y)=0$ for all $y \in D$, which in turn implies that $x=0$ as $\operatorname{Trd}_{D / Z}$ is an involution trace. Thus $s$ satisfies the third condition as well for being an involution trace. 
Now, applying Proposition 4.7 in [KMRT98] to $E=D, T=F$ and $M=D^{n}$, we get a nonsingular hermitian or skew hermitian form (according as $h$ ) with respect to $\theta$ on $F$. The nonsingular form can be written as the following:

$$
s_{*}(h): D^{n} \times D^{n} \rightarrow F
$$

such that

$$
s_{*}(h)(x, y)=s(h(x, y)) \text { for } x, y \in D^{n} .
$$

Also, $D^{n}$ is a right vector space over $F$ and $\operatorname{End}_{D}\left(D^{n}\right) \subset \operatorname{End}_{F}\left(D^{n}\right)$. The adjoint involution $\sigma_{s_{*}(h)}$ on $\operatorname{End}_{F}\left(D^{n}\right)$ extends the adjoint involution $\sigma=\sigma_{h}$ on $\operatorname{End}_{D}\left(D^{n}\right)$, that is,

$$
\left(\operatorname{End}_{D}\left(D^{n}\right), \sigma_{h}\right) \subset\left(\operatorname{End}_{F}\left(D^{n}\right), \sigma_{s_{*}(h)}\right)
$$

In view of the above discussion Eq. 3.1 can be written as:

$$
\begin{aligned}
(F G, \sigma) \simeq \Pi_{i=1}^{r}\left(\operatorname{End}_{D_{i}}\left(D_{i}^{a_{i}}\right), \sigma_{a_{i}}\right) \times \\
\Pi_{j=1}^{t}\left(\operatorname{End}_{D_{j}^{\prime} \times D_{j}^{\prime o p}}\left(D_{j}^{\prime b_{j}} \times\left(D_{j}^{\prime o p}\right)^{b_{j}}\right), \sigma_{b_{j}}\right) \\
\subset \Pi_{i=1}^{r}\left(\operatorname{End}_{F}\left(D_{i}^{a_{i}}\right), \sigma_{s_{*}\left(h_{a_{i}}\right)}\right) \times \\
\Pi_{j=1}^{t}\left(\operatorname{End}_{F \times F}\left(D_{j}^{\prime b_{j}} \times\left(D_{j}^{\prime o p}\right)^{b_{j}}, \sigma_{s_{*}\left(h_{b_{j}}\right)}\right),\right.
\end{aligned}
$$

where $\sigma_{a_{i}}=\sigma_{h_{a_{i}}}$ are the adjoint involutions of the nonsingular hermitian or skew hermitian forms $h_{a_{i}}: D_{i}^{a_{i}} \times D_{i}^{a_{i}} \rightarrow D_{i}$ with respect to the involution $\theta_{a_{i}}$ (which is of the same kind as $\sigma_{h_{a_{i}}}$ ) on $D_{i}$ and $\sigma_{b_{j}}=\sigma_{h_{b_{j}}}$ are the adjoint involutions of the nonsingular hermitian forms $h_{b_{j}}:\left(D_{j}^{\prime b_{j}} \times\left(D_{j}^{\prime o p}\right)^{b_{j}}\right) \times\left(D_{j}^{\prime b_{j}} \times\left(D_{j}^{\prime o p}\right)^{b_{j}}\right) \rightarrow D_{j}^{\prime} \times D_{j}^{\prime o p}$ with respect to the involution (of second kind) $\theta_{b_{j}}$ on $D_{j}^{\prime} \times D_{j}^{\prime o p}$.

The set $\left\{x \in \operatorname{End}_{D}\left(D^{n}\right) \mid h(x u, v)+h(u, x v)=0\right.$ for all $\left.u, v \in D^{n}\right\}$, where $h$ : $D^{n} \times D^{n} \rightarrow D$ is a nonsingular hermitian or skew-hermitian form on $D^{n}$ with respect to some involution $\theta$ (of any kind) on $D$, is a Lie algebra over $F$.

Now, $D^{n}$ is a simple $F G$-module. Thus each $f \in F G$ acts on $D^{n}$. Thus the projection, say $f_{i}$ of $f$ on any of the factors $M_{n}(D) \simeq \operatorname{End}_{D}\left(D^{n}\right)$ of $F G$ can be viewed as an element in $\operatorname{End}_{D}\left(D^{n}\right)$. Now we prove the following characterization of the projection of the skew-symmetric elements on one of the summands of $F G$.

Lemma 3.3. The image of $F G_{\sigma}^{-}$under the projection of $F G$ onto $M_{n}(D)$ consists of all linear transformations $f_{i}$ in $\operatorname{End}_{D}\left(D^{n}\right)$ such that $s_{*}(h)\left(f_{i}(x), y\right)+s_{*}(h)\left(x, f_{i}(y)\right)=0$ for all $x, y \in D^{n}$. 
Proof. Recall that $\sigma=\sigma_{h}$ is the adjoint involution of the nonsingular hermitian or skew-hermitian form $h$ on $D^{n}$ with respect to $\theta$, that is, $h(x, f(y))=h\left(\sigma_{h}(f)(x), y\right)$ for all $x, y \in D^{n}$ and $f \in \operatorname{End}_{D}\left(D^{n}\right)$. If $f_{i}$ belongs to the image of $F G_{\sigma}^{-}$under the projection of $F G$ onto $M_{n}(D)$, then $\sigma\left(f_{i}\right)=-f_{i}$, by definition of $F G_{\sigma}^{-}$. The involution $\sigma_{s_{*}(h)}$ of $\operatorname{End}_{F}\left(D^{n}\right)$ extends the involution $\sigma_{h}$ on $\operatorname{End}_{D}\left(D^{n}\right)$. That is, $\sigma_{s_{*}(h)}$ restricted to $\operatorname{End}_{D}\left(D^{n}\right)$ is $\sigma_{h}$. Thus, as $f_{i} \in \operatorname{End}_{D}\left(D^{n}\right)$, we have $\sigma_{s_{*}(h)}\left(f_{i}\right)=\sigma_{h}\left(f_{i}\right)=-f_{i}$. This implies $h\left(x, f_{i}(y)\right)=h\left(\sigma_{h}\left(f_{i}\right)(x), y\right)=-h\left(f_{i}(x), y\right)$. So we get

$$
\sigma\left(f_{i}\right)=-f_{i} \Leftrightarrow s_{*}(h)\left(f_{i}(x), y\right)+s_{*}(h)\left(x, f_{i}(y)\right)=0 \text { for } x, y \in D^{n} .
$$

We are now in a position to prove our main theorems. Recall definitions 2.4 and 2.5 for the proof of Theorem 1.1 .

Proof of Theorem 1.1. Let $M=\Pi_{i=1}^{r} D_{i}^{a_{i}} \times \Pi_{j=1}^{t}\left(D_{j}^{\prime b_{j}} \times\left(D_{j}^{\prime o p}\right)^{b_{j}}\right)$. Then $M$ is a right vector space over $F$ and also a left $F G$-module. Define $h: M \times M \rightarrow F$ by

$$
h\left(\sum_{i=1}^{r} x_{i}+\sum_{j=1}^{t} x_{j}^{\prime}, \sum_{i=1}^{r} y_{i}+\sum_{j=1}^{t} y_{j}^{\prime}\right)=\bigoplus_{i=1}^{r} s_{*}\left(h_{a_{i}}\right)\left(x_{i}, y_{i}\right) \oplus \bigoplus_{j=1}^{t} s_{*}\left(h_{b_{j}}\right)\left(x_{j}^{\prime}, y_{j}^{\prime}\right),
$$

where $x_{i}, y_{i} \in D_{i}^{a_{i}}$ and $x_{j}^{\prime}, y_{j}^{\prime} \in D_{j}^{\prime b_{j}} \times\left(D_{j}^{\prime o p}\right)^{b_{j}}$. Then $h$ is a nonsingular form (hermitian or skew-hermitian) on $M$ with respect to the involution $\theta$ on $F$, where $\theta=$ $\bigoplus_{i=1}^{r} \theta_{a_{i}} \oplus \bigoplus_{j=1}^{t} \theta_{b_{j}}$ with $\theta_{a_{i}}$ being $F$-restrictions of the corresponding involutions on $D_{i}$ for $1 \leq i \leq r$ and $\theta_{b_{j}}$ being $F \times F$-restrictions of the corresponding involutions on $D_{j}^{\prime} \times D_{j}^{\prime o p}$ for $1 \leq j \leq t$. Also we have the given involution $\sigma$ on $F G$ is the adjoint involution of $h$ as by construction $\sigma$ is expressed as the sum of adjoint involutions on each simple component of $F G$ with respect to the corresponding nonsingular hermitian or skew-hermitian form on that component. With the above notations, we will show that Equation 1.2 of Theorem 1.1 holds.

Let $f \in F G_{\sigma}^{-} \subset(F G, \sigma)$. Thus $f=\sum_{i=1}^{r} f_{i}+\sum_{j=1}^{t} f_{j}^{\prime}$, where each $f_{i}$ (resp. $f_{j}^{\prime}$ ) belongs to the image of $F G_{\sigma}^{-}$under the projection of $F G$ onto $\operatorname{End}_{D_{i}}\left(D_{i}^{a_{i}}\right)$ (resp. $\left.\operatorname{End}_{D_{j}^{\prime} \times D_{j}^{\prime o p}}\left(D_{j}^{\prime b_{j}} \times\left(D_{j}^{\prime o p}\right)^{b_{j}}\right)\right)$. Writing $x=\sum_{i=1}^{r} x_{i}+\sum_{j=1}^{t} x_{j}^{\prime}$ and $y=\sum_{i=1}^{r} y_{i}+$ $\sum_{j=1}^{t} y_{j}^{\prime}$, and using Lemma 3.3, we have:

$$
\sigma(f)=-f
$$




$$
\begin{array}{ll} 
& \Longleftrightarrow \quad \bigoplus_{i=1}^{r} \sigma_{h_{a_{i}}}\left(f_{i}\right) \oplus \bigoplus_{j=1}^{t} \sigma_{h_{b_{j}}}\left(f_{j}^{\prime}\right)=-\sum_{i=1}^{r} f_{i}-\sum_{j=1}^{t} f_{j}^{\prime} \\
& \Longleftrightarrow \quad \bigoplus_{i=1}^{r}\left(s_{*}\left(h_{a_{i}}\right)\left(f_{i}\left(x_{i}\right), y_{i}\right)+s_{*}\left(h_{a_{i}}\right)\left(x_{i}, f_{i}\left(y_{i}\right)\right)\right) \\
& \oplus \bigoplus_{j=1}^{t}\left(s_{*}\left(h_{b_{j}}\right)\left(f_{j}^{\prime}\left(x_{j}^{\prime}\right), y_{j}^{\prime}\right)+s_{*}\left(h_{b_{j}}\right)\left(x_{j}^{\prime}, f_{j}^{\prime}\left(y_{j}^{\prime}\right)\right)\right)=0 \\
& h(f(x), y)+h(x, f(y))=0 .
\end{array}
$$

Proof of Theorem 1.2. It follows from Lemma 3.3 that the image of $F G_{\sigma}^{-}$under the projection of $F G$ onto $M_{n}(D)$ is a Lie algebra. Let $V=D^{n}$. Then $\mathrm{V}$ is an irreducible left FG-module. From Theorem 4.2, [KMRT98] it follows that if $\sigma$ is of the first kind on $\operatorname{End}_{D}(V)$, then $\sigma$ restricted to $\operatorname{End}_{D}(V)$ is orthogonal (resp. symplectic) if the involution $\theta$ on $D$ is orthogonal (resp. symplectic) provided the corresponding nonsingular form $h: V \times V \rightarrow D$ with respect to $\theta$ on $D$ is hermitian. If the nonsingular form $h$ is skew-hermitian, then $\sigma$ restricted to $\operatorname{End}_{D}(V)$ is orthogonal (resp. symplectic) if $\theta$ is symplectic (resp. orthogonal). Let the degree of the central simple algebra $\operatorname{End}_{D}(V)$ over its center $Z$ be $s$. By Proposition 2.6 and Proposition 2.17 of [KMRT98] the dimension of the Lie algebra $\operatorname{End}_{D}(V)_{\sigma}^{-}$is $s(s-1) / 2$ if $\sigma$ restricted to it is orthogonal and the dimension is $s(s+1) / 2$ if $\sigma$ restricted to it is symplectic. Whenever the involution $\sigma$ is symplectic, $s$ is even. If $\sigma$ is of the second kind (that is of unitary type) on $\operatorname{End}_{D}(V)$ or $E n d_{D \times D^{o p}}\left(V \times V^{o p}\right)$, then the dimension is $s^{2}$. Denoting the Lie algebras $\operatorname{End}_{D}(V)_{\sigma}^{-}$in the orthogonal, symplectic and unitary cases as $\mathfrak{o}(V), \mathfrak{s p}(V)$ and $\mathfrak{g l}(V)$ respectively, we get the decomposition structure of $F G_{\sigma}^{-}$as proposed.

\section{ReFERENCES}

[Ami69] S.A. Amitsur. Identities in rings with involutions. Israel J. Math., 7:63-68, 1969.

[BGBT18] K.J. Becher, N. Grenier-Boley, and J.P. Tignol. Involutions and stable subalgebras. J. Algebra, 493:381-409, 2018.

[CJMM09] O.B. Cristo, E. Jespers, C.P. Milies, and M. Ruiz Marín. Antisymmetric elements in group rings II. J. Algebra Appl., 8:115-127, 2009.

[CM07] O.B. Cristo and C.P. Milies. Commutativity of skew elements in group rings. Proc. Edinburgh Math. Soc., 50:37-47, 2007.

[CT07] A.M. Cohen and D.E. Taylor. On a certain Lie algebra defined by a finite group. Amer. Math. Monthly, 114:633-639, 2007.

[GM03] A. Giambruno and C.P. Milies. Unitary Units and skew elements in group algebras. Manuscripta Math., 111:195-209, 2003.

[GS93] A. Giambruno and S.K. Sehgal. Lie nilpotence of group rings. Comm. Algebra, 21:42534261, 1993. 
[JM05] E. Jespers and M. Ruiz Marín. Antisymmetric elements in group rings. J. Algebra Appl., 4:341-353, 2005.

[JM06] E. Jespers and M. Ruiz Marín. On symmetric elements and symmetric units in group rings. Comm. Algebra, 34:727-736, 2006.

[KMRT98] M.A. Knus, A. Merkurjev, M. Rost, and J.P. Tignol. The Book of Involutions. Colloquium Publications, Vol. 44, American Mathematical Society, 1998.

[Knu91] M.A. Knus. Quadratic and Hermitian Forms over Rings. Grundlehren der Mathematischen Wissenschaften, Vol. 294, Springer, 1991.

[LSS09] G.T. Lee, S.K. Sehgal, and E. Spinelli. Group algebras whose symmetric and skew elements are Lie solvable. Forum Math., 21:661-671, 2009.

[Mar10] I. Marin. Group algebras of finite groups as Lie algebras. Comm. Algebra, 38:2572-2584, 2010.

[Rei03] I. Reiner. Maximal Orders. London Mathematical Society Monographs New Series, Vol. 28, The Clarendon Press, Oxford University Press, 2003.

[ZS81] A.E. Zaleskii and M.B. Smirnov. Lie algebra associated with linear group. Comm. Algebra, 9:2075-2100, 1981.

Department of Mathematical Sciences, Indian Institute of Science Education and ReSearch Mohali, Sector-81, Knowledge City, S.A.S. Nagar, Mohali-140306, Punjab, InDIA

E-mail address: dishari@iitg.ac.in, dishari.chaudhuri@gmail.com 